\title{
3.4 OBSERVATIONS OF PULSAR SPECTRA
}

\author{
M. S. EWING, R. A. BATCHELOR*, R. FRIEFELD, R. M. PRICE, \\ and D. H.STAELIN
}

Research Laboratory of Electronics, Massachusetts Institute of Technology, Cambridge, Mass., U.S.A.

\begin{abstract}
The observed width of spectral features in CP 0328, CP 0834, and CP 1919 are approxmately proportional to the fourth power of frequency, thus supporting the hypothesis that the slow spectral variations of these pulsars are due to interstellar scintillation. The spectral features in CP 0834, CP 1133, and CP 1919 are observed to drift systematically at rates compatible with a simple interstellar scintillation model. Pulsar velocities of $\sim 100 \mathrm{~km} \mathrm{sec}^{-1}$ are inferred from these spectral drift rates.
\end{abstract}

\section{Introduction}

Observations of pulsar spectra yield information about the intrinsic radiation properties of pulsars, and about the intervening interstellar medium. In an effort to separate and study these two aspects of pulsar spectra, we have observed since 1968 the spectra of those pulsars within the range of the National Radio Astronomy Observatory 300-ft transit telescope in Green Bank, West Virginia. The spectrometers each had 50 channels with bandwidths of 100,30 , or $10 \mathrm{kHz}$, or had 40 channels with bandwidths of $1 \mathrm{MHz}$. The center frequencies of the filter banks ranged from 110 to $550 \mathrm{MHz}$, and all 40 or 50 channels were sampled with 8-bit accuracy every $30 \mathrm{msec}$. Subsequent computer analysis yielded the spectra of individual and average pulses. Additionally, in May, 1970 , both the $300-\mathrm{ft}$ transit telescope and the 140-ft fully steerable telescope in Green Bank were used in conjunction with the 384-channel autocorrelation receiver to achieve frequency resolution as high as $1 \mathrm{kHz}$.

The present report describes the results of manual analyses of the spectra of four pulsars, CP 0328, CP 0834, CP 1133, and CP 1919. A more complete analysis of all the spectral data is in preparation.

\section{Widths of Spectral Features}

Typical data obtained with the multichannel filter systems are presented in Figure 1. These data were processed by computer, displayed on a cathode-ray-tube, and then photographed. Each resolution element in these photographs represents the average of several pulses within a single channel. The three light levels represent relative pulse energy thresholds of 1,2 , and 4 . The figure illustrates how the spectral features in each pulsar develop and change.

One parameter of interest in these spectra is the frequency dependence of spectral feature width. Feature widths were determined by averaging the visually determined instantaneous full widths at half-maximum, $B$, for a large number of spectral features at each frequency. Typical feature widths observed using the autocorrelator for these

* On leave from Division of Radiophysics, CSIRO, Sydney, Australia. 

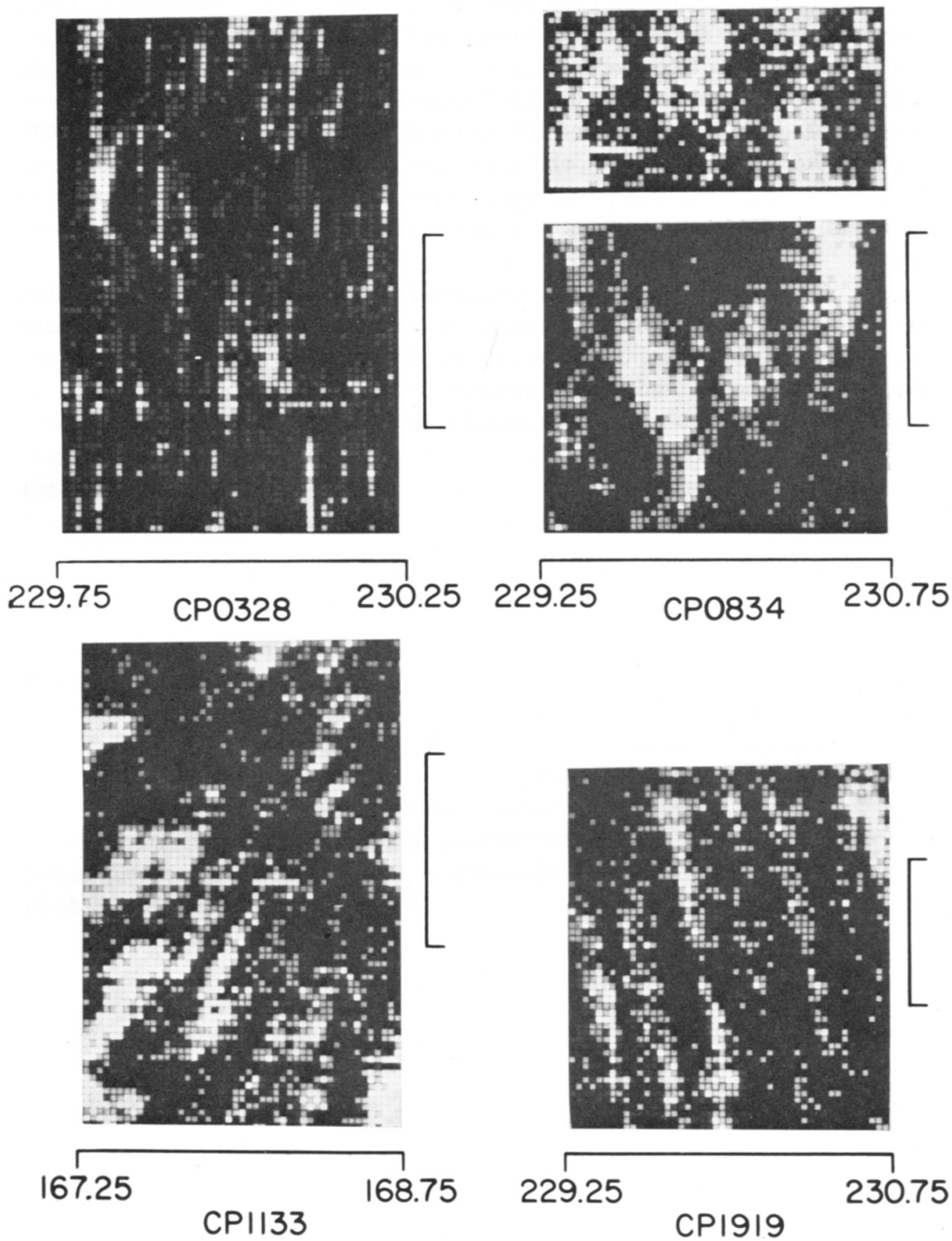

Fig. 1. Representative dynamic spectra for CP 0328, CP 0834, CP 1133, and CP 1919 as observed with 50-channel spectrometers. The light levels represent relative power thresholds of 1,2 , and 4, in order of increasing brightness. Time increases from top to bottom, and the scale markers indicate 10 minutes. 
four pulsars are listed in Table I as a function of frequency and are plotted in Figure 2. The feature widths $B$ are assumed to vary as $f^{\alpha}$. Least squares fits for $\alpha$ yield the numbers in Table I. The errors quoted are estimated maximum deviations from the mean fit. The three pulsars excluding CP 1133 are fairly consistent with a value of $\alpha=4$. An $f^{4}$ dependence is generally consistent with the results reported by Rickett (1969), Staelin (1969), and Lang (1970), and apparently inconsistent with those reported by Huguenin et al. (1969). Some of the widths reported here are less than those reported by others, possibly because a cluster of narrow features can resemble a single broad feature.

An $f^{4}$ dependence of feature width is predicted by scintillation theory. In particular, the $f^{4}$ dependence follows if we assume the antenna intercepts rays which have traversed different paths, and that the path lengths differ by a nominal value $\delta$, where $\delta$ arises geometrically, and is approximately $R \theta_{\mathrm{s}}^{2} / 2$, where $R$ is the distance to the pulsar, and $\theta_{s}$ is the rms angle of ray arrival at the antenna. This same model predicts
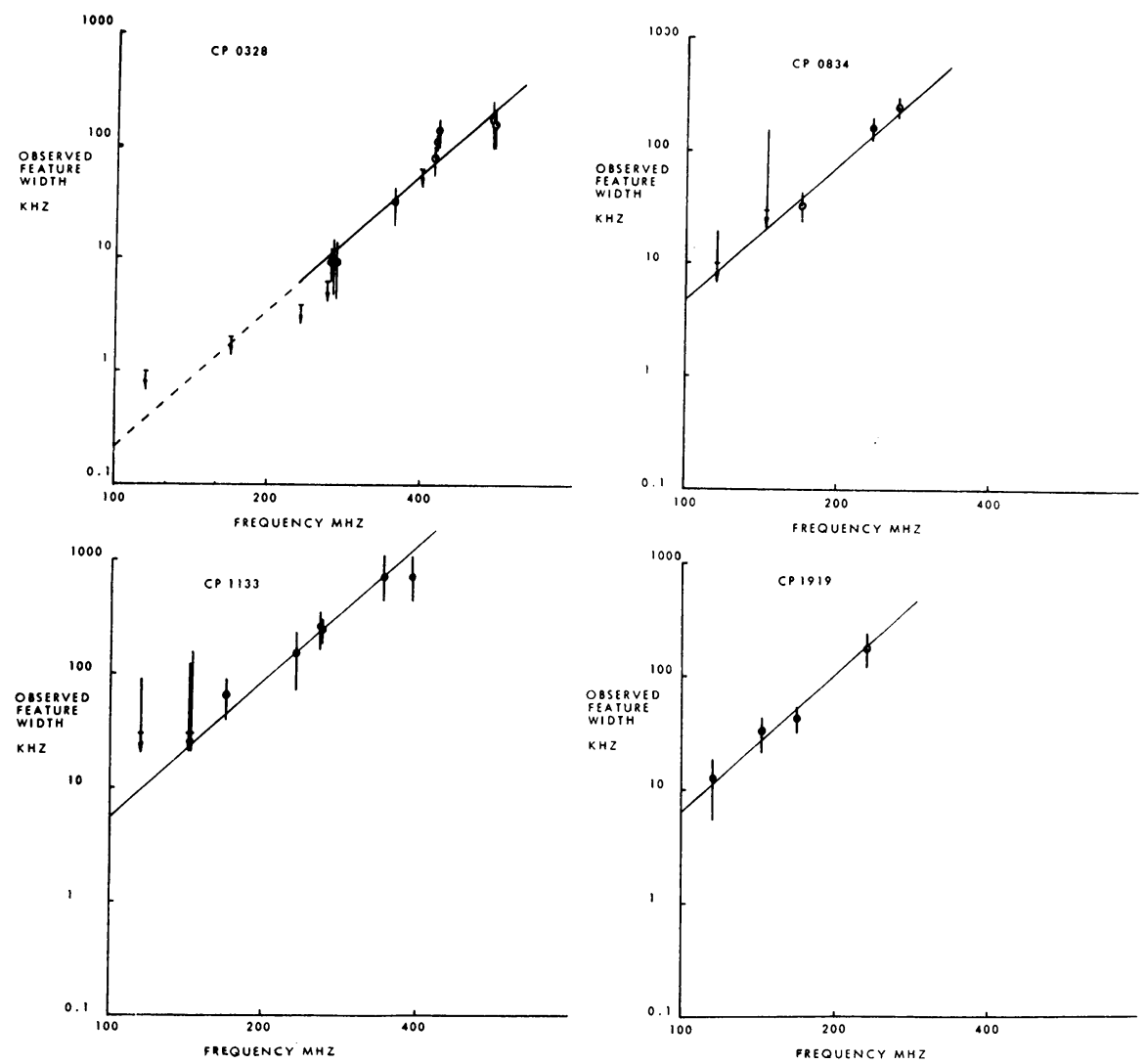

Fig. 2. Wavelength dependence of spectral feature widths. The widths represent averages of many spectral features, and the error bars indicate the total range of observed feature widths. Arrows and cross-bars represent the limitations of spectral resolution. The sloping lines correspond to a $f^{4}$ dependence of feature width. 
TABLE I

Pulsar spectral feature widths

\begin{tabular}{llllllll}
\hline Source & Freq $(\mathrm{MHz})$ & $B(\mathrm{kHz})$ & $\alpha$ & Source & Freq $(\mathrm{MHz})$ & $B(\mathrm{kHz})$ & $\alpha$ \\
\hline CP 0328 & 112 & $<1 ?$ & & CP 1133 & 112 & $<90$ & \\
& 168 & $\leqslant 2$ & & 142 & $<150$ & \\
& 267 & $10 \pm 5$ & & 168 & $65 \pm 25$ & \\
& 350 & $33 \pm 13$ & & 230 & $150 \pm 80$ & \\
& 405 & $111 \pm 50$ & & 350 & $417 \pm 70$ & \\
& 560 & $170 \pm 70$ & $3.9 \pm 0.8$ & & 405 & $692 \pm 250$ & $2.6 \pm 0.6$ \\
CP 0834 & 112 & $<20$ & & CP 1919 & 112 & $13 \pm 7$ & \\
& 142 & $<150$ & & 142 & $34 \pm 10$ & \\
& 168 & $34 \pm 9$ & & 168 & $38 \pm 20$ & \\
& 230 & $162 \pm 35$ & & & $178 \pm 60$ & $3.5 \pm 1.1$ \\
& 258 & $245 \pm 40$ & $4.6 \pm 0.5$ & & & \\
\hline
\end{tabular}

that the spectral feature widths will also be approximately inversely proportional to the square of the dispersion measure, a result compatible with the present observations. Such scintillation models have been discussed by Scheuer (1968), Salpeter (1969), and Uscinski (1968a, b).

In the case of the Crab Nebula pulsar, NP 0531, the value of $\delta$ and its $f^{-4}$ dependence has been measured directly by Staelin and Sutton (1970), and Rankin et al. (1970). Using the observed value of $13 \mathrm{msec}$ for the multipath delay at $115 \mathrm{MHz}$, this model predicts feature widths for NP 0531 of $\sim 2 \mathrm{kHz}$ near $300 \mathrm{MHz}$. This is too narrow to be observed readily, and may account for the apparent lack of spectral features in the Crab Nebula pulsar. This feature width is narrower than those of other pulsars scaled by the inverse square of the dispersion measure, but is within a factor of 8 of the expected value, and within a factor of 2 of values extrapolated from CP 0328 and CP 1133. It is therefore possible that the general interstellar medium, rather than the pulsar environment, is responsible for the multipath effects and lack of observable scintillation in NP 0531 .

\section{Drifting of Spectral Features}

A very interesting property of some pulsars is systematic drifting of spectral features, as illustrated for CP 1919 and CP 1133 in Figure 1. Drifting has been observed in each of these two pulsars on several occasions, although the phenomenon is readily overlooked if the spectrometer resolution is not appropriate, or if the operating frequency is such that the drift rate is too slow or obscured by variations in intrinsic pulsar intensity. Drifting has also probably been observed in CP 0834. Representative observed drift rates for CP 0834, CP 1133, and CP 1919 are $130 \mathrm{~Hz} \mathrm{sec}^{-1}$ at $168 \mathrm{MHz}$, $1 \mathrm{kHz} \mathrm{sec}-1$ at $230 \mathrm{MHz}$, and $500 \mathrm{~Hz} \mathrm{sec}-1$ at $230 \mathrm{MHz}$, respectively. The drift rates appear to vary from month to month, and may vary on shorter time scales. The drifts of both CP 1919 and CP 1133 have changed directions, and at times different simultaneous spectral features may have different drift rates. 
A simple model for interstellar scintillation yields an understanding of spectral drifting. We may assume that the radiation propagating from the pulsar to Earth is composed of rays, each executing a random walk characterized by $\theta_{s}$, the rms angle between any ray segment and the direct path. These rays converge at the Earth with different arrival angles and different delays. If many scattering events occur for each ray, then the propagation delay and the angle of arrival may be weakly correlated, in contrast to the single thin-screen model. Consider the case where the radiation incident upon the Earth is dominated by two rays. The interference of the rays produces an interference pattern through which the Earth moves at velocity $v_{0}$. In this case the lifetime $\Delta t$ of a single spectral feature, i.e. the time between half-power points at a single frequency, is the time required for the Earth to move past one lobe of the interference pattern, i.e.

$$
\Delta t \cong \frac{\lambda}{\sqrt{8} \theta_{s} v_{0}}
$$

where $\lambda$ is the wavelength, and $\sqrt{8}$ is an approximate geometrical factor. A frequency drift can result if the propagation delays for these two rays are different. The phenomenon is analogous to the movement of an observer through the frequencydependent lobes of a transmitting interferometer. If the delays differ by the reasonable value $R \theta_{s}^{2} / 2$, where $R$ is the pulsar-Earth separation, then the feature drift rate $f$ is

$$
f \cong f v_{0} \sqrt{\frac{8 B}{R c}} .
$$

Since the nominal width $B$ is proportional to $f^{4} R^{-2}$, it follows that the drift rate $f$ should be proportional to $f^{3} R^{-3 / 2}$. Consistency of these expressions requires that $f \cong B / \Delta t$, where $B \cong c / R \theta_{s}^{2}$.

By averaging the drift rates of several spectral features it is possible to estimate the magnitude and frequency dependence of the drift rates. Since rays of different frequencies have different interstellar propagation paths, and since the paths are time varying, many observations will be required. For the present limited data the drift rate is proportional to $f^{3 \pm 1.5}$. Although the observations are consistent with the $R^{-3 / 2}$ dependence upon pulsar distance, the results are not definitive. The drift rate predicted for CP 0328 is approximately $70 \mathrm{~Hz} \mathrm{sec}^{-1}$ at $200 \mathrm{MHz}$, which is too small to be evident in the spectra of Figure 1.

The observations of $f$ can yield an independent estimate of the transverse pulsar velocity $v$. We deduce approximate velocities of $100 \mathrm{~km} \mathrm{sec}^{-1}$ for all three pulsars using the drift rate, bandwidths, and equations cited earlier, and assuming the interstellar electron density is $0.03 \mathrm{~cm}^{-3}$ (Staelin and Reifenstein, 1968). This equals the velocities deduced from the feature lifetimes $\Delta t$. For example, for the data of Figure 1 and Table I, the approximate velocity formula

$$
v \cong \sqrt{\frac{c R B}{8} \frac{1}{v \Delta t}}
$$


yields for CP 0328, CP 0834, CP 1133, and CP 1919 velocities of $100 \mathrm{~km} \mathrm{sec}^{-1}$, within a factor of 2. Rickett (1969) and Lang (1970) have deduced similar velocities from their feature-lifetime data. Further corroboration follows from the measurements by Lang and Rickett (1970) of scintillation delay between spectral features observed at Arecibo and Jodrell Bank, which yielded velocities for CP 1133 of approximately $100 \mathrm{~km} \mathrm{sec}^{-1}$.

Since the velocity of the Earth with respect to the interstellar medium within $2 \mathrm{kpc}$ is generally less than $50 \mathrm{~km} \mathrm{sec}^{-1}$, these data suggest that the average transverse velocity of these three pulsars with respect to the interstellar medium may be of the order of $100 \mathrm{~km} \mathrm{sec}^{-1}$, which is consistent with the velocities of runaway stars (Gott et al., 1970; Gunn and Ostriker, 1970; and Prentice, 1970) and of NP 0531. More extensive drift rate data and more accurate theoretical analysis could further strengthen this conclusion.

The consistency of these spectral observations with theoretical models of interstellar scintillation further supports suggestions of Rickett (1969) and others that the observed slow spectral changes originate in the interstellar medium. Our observations have extended this conclusion to spectral features of widths ranging from $2 \mathrm{kHz}$ to several $\mathrm{MHz}$.

\section{Acknowledgements}

We thank J. Sutton for helpful conversations and we acknowledge the cooperation and assistance of W. Brundage, J. Greenhalgh, and other staff of the National Radio Astronomy Observatory, which is operated by Associated Universities, Inc., under contract with the National Science Foundation.

The work of M. S. Ewing and R. M. Price was supported principally by National Science Foundation grant GP13056, and that of D. H. Staelin was supported principally by grant GP14854.

\section{References}

Gott, J. R., III, Gunn, J. E., and Ostriker, J. P.: 1970, Astrophys. J. 160, L91.

Gunn, J. E. and Ostriker, J. P.: 1970, Astrophys. J. 160, 979.

Huguenin, G. R., Taylor, J. H., and Jura, M.: 1969, Astrophys. Letters 4, 71.

Lang, K. R.: 1970, 'Interstellar Scintillation of Pulsar Radiation', preprint.

Lang, K. R. and Rickett, B. J.: 1970, Nature 225, 528.

Prentice, A. J. R.: 1970, Nature 225, 438.

Rankin, J. M., Comella, J. M., Craft, H. D., Jr., Richards, D. W., Campbell, D. B., and Counselman, C. C., III: 1970, Astrophys. J. 162, 707.

Rickett, B. J.: 1969, Nature 221, 158.

Rickett, B. J.: 1970, Monthly Notices Roy. Astron. Soc. 150, 67.

Salpeter, E. E.: 1969, Nature 221, 31.

Scheuer, P. A. G.: 1968, Nature 218, 920.

Staelin, D. H.: 1969, contribution to Accademia Nazionale Dei Lincei meeting on 'Pulsars and High Energy Activity in Supernovae Remnants'.

Staelin, D. H. and Reifenstein, E. C., III: 1968, Science 162, 1481.

Staelin, D. H. and Sutton, J. M.: 1970, Nature 226, 69.

Uscinski, B. J.: 1968a, Phil. Trans. A262, 609.

Uscinski, B. J.: 1968b, Proc. Roy. Soc. A307, 471. 